\title{
Effect of Correlating ImageThreshold Values with Image Gradient Field on Damage Detection in Composite Structures
}

\author{
Mahmoud Zaki Iskandarani \\ Faculty of Engineering \\ Al-Ahliyya Amman University, Amman-Jordan
}

\begin{abstract}
Effect of image threshold level variation is studied and proved to be a critical factor in damage detection and characterization of impacted composite Reaction Injection Molding ((RIM) structures. The variation of threshold is used as an input to both gradient field algorithm and segmentation algorithm. The choice of optimum threshold for a tested composite type is achieved as a result of correlation between the resulted gradient field images and segmented images. Type and extent of damage is also analyzed using detailed pixel distribution as a function of both impact energy and threshold level variation. The demonstrated cascading based technique is shown to be promising for an accurate testing and classification of damage in composite structures in many critical areas such as medical, aerospace and automotive.
\end{abstract}

Keywords-Gradient norm; edge detection; gray level mapping; segmentation; threshold; histogram; image processing; composites; impact damage

\section{INTRODUCTION}

Testing composite structures is one of the most important tasks employed in maintenance and diagnosis of components. Non-Destructive Testing (NDT) techniques are used in the aircraft design, automotive design as well as many other applications. Applications for composite materials has expanded from the use as a non-structural part to the construction of complete frames and body structures due to its toughness, stiffness and high strength to weight ratio.

A composite structure is normally made up of several plies with selected fiber orientation and characteristics. The impact damage of a composite laminate can lead to more damage than it is apparent on the surface. Repetitive impact loading can result in severe damage, which would end in component structural failure. Cracks and debonding (delamination) are common damage cases found in composite structures. Delamination is more critical as it causes reduction the loadcarrying capability of a composite component, resulting in catastrophic failure.

Images (Thermal, Ultrasonic, and Visual) for damaged composites will most of time show segmentation. Thus subdivides the composite image into regions. The number of regions formed as a result of segmentation is a function of damage type. The segmented image is a function of both discontinuity and uniformity, which are used to determine similarity and damage magnitude [1-10].
Image segmentation is considered to be vital in analyzing an acquired image in many computer image processing applications such as medical imaging, robotic vision, face recognition applications and many others. Segmentation will partition an image into foreground and background with respect to selected variables. Most of image segmentation techniques are based similarity and discontinuity. In addition to used segmentation methods, thresholding is regarded as a major contributor to achieve accurate image interpretation and subsequent classification, as histogram related thresholding is complex especially for multi-level thresholding [11-15]. It is critical for damage detection in composite structures to implement image enhancement techniques which is essential in image processing with particular consideration to intensity variation of image contents and its effect on segmentation.

\section{BACKGROUND}

Detecting damage in composite structures is a challenging task owing to the variable appearance and the wide range of shapes and textures that composites can exhibit. The first need is a reliable feature set that allows the damage type to be distinguished clearly, especially in shadowed backgrounds and under difficult illumination. Image segmentation is a critical and challenging problem in image processing and is the initial step for high level analysis. The objective behind image segmentation is to divide an image into different classes based on features, such as color, intensity or histogram, such that pixels are grouped under different classes.

Image segmentation is used to divide an image into multiple segments in order to obtain data that can be used for classification and decision making. The purpose of dividing an image into different region is to enable further image analysis.

Image segmentation has been widely used in many practical applications such as medical imaging, remote sensing, Optical Character Recognition (OCR) and general object detection. Segmentation will transform representation into something that is more representative to study or examine. Image segmentation is mainly used to detect objects and background in images. More specifically, image segmentation is the mechanism of assigning a label to each pixel in an image such that pixels with the same tag share certain characteristics.

The core of image segmentation is thresholding, which can be used to generate binary images by selecting optimum 
threshold value for accurate segmentation. Many conventional methods are used in engineering including the $\mathrm{k}$-means clustering, Otsu's method, which employ maximum entropy and maximum variance method. Other related techniques for feature extraction and object recognition, such as Histogram of Gradients (HOG) and wavelet transform are also used [1619].

Segmentation can be more effective if an optimum level of thresholding is achieved, thus, more accurate interpretation of acquired images and better analysis are obtained, resulting in a well-defined region-based description of images through decomposing them into $\mathrm{r}$ spatially coherent regions sharing similar attributes.

In this work, a new approach to damage detection and composite structure analysis is applied to images of impact damaged composites. The technique optimizes and localizes the boundaries of a damaged area as a function of image gradient and threshold values, which is then correlated with impact energies. The approach uses segmentation for both colour and grey images and eliminates histogram intensity variation with image contents by using histogram gradients.

\section{MATERIAL AND METHODS}

The tested RIM composites subjected to Impact Energies $\{0,14 \mathrm{~J}, 28 \mathrm{~J}, 42 \mathrm{~J}, 56 \mathrm{~J}\}$, hence, increments of $14 \mathrm{~J}$. Up to $28 \mathrm{~J}$ Impact Energy, no evidence of damage was realized. Thus, the considered Impact energies in this work are $\{28 \mathrm{~J}, 42 \mathrm{~J}, 56 \mathrm{~J}\}$.

The main objective is to produce an automatic damage characterization and classification through thresholding and image segmentation. Thus, obtaining a region-based description of the damage embedded in an image by dividing the image it into spatially coherent regions with similar attributes.

To achieve the intended purpose of this work, images of damaged structures at various impact energies are tested using MATLAB algorithm as shown in Fig. 1.

Two or more sampling points are considered at non-zero grayscale levels when:

- All of the levels are empty

- All of the levels are full

Sampling stops at the first threshold level where no segmentation is possible. The threshold level just before the one that caused a no segmentation condition is considered the optimum threshold level for a composite type and impact energy level. Any condition outside these is classified as total damage and matrix-fiber failure.

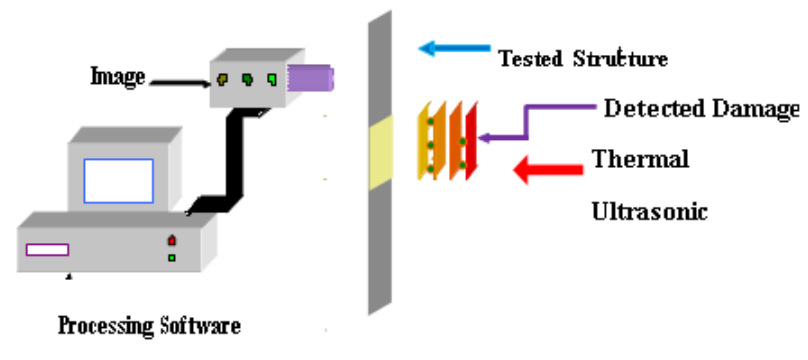

Fig. 1. Experimental Setup.

\section{RESULTS}

\section{A. 28J Impact Energy}

Table I shows t effect of varying the threshold level on the pixel distribution of an impact damaged RIM composite structure. The structure suffered a 28J of impact energy delivered through a vertically launched load, while Fig. 2-9 shows both Gradient Field and Segmentation images.

TABLE I. PIXEL DisTRIBUTION FOR 28J IMPACT

\begin{tabular}{|c|c|c|c|c|}
\hline Threshold & Level 0 & Level 128 & Level 180 & Level 255 \\
\hline 0.42 & 14440 & 0 & $\mathbf{0}$ & 0 \\
\hline 0.43 & 14396 & 4 & 0 & 0 \\
\hline 0.44 & 14378 & 18 & 4 & $\mathbf{0}$ \\
\hline 0.45 & 14362 & 30 & 8 & 0 \\
\hline 0.46 & 14328 & 58 & 14 & $\mathbf{0}$ \\
\hline 0.47 & 14320 & 60 & 20 & $\mathbf{0}$ \\
\hline 0.48 & 14300 & 68 & 32 & $\mathbf{0}$ \\
\hline 0.49 & 14290 & 68 & 42 & 0 \\
\hline 0.50 & 14252 & 92 & 56 & 0 \\
\hline 0.51 & 14156 & 184 & 60 & $\mathbf{0}$ \\
\hline 0.52 & 14096 & 222 & 82 & $\mathbf{0}$ \\
\hline 0.53 & 14002 & 272 & 126 & $\mathbf{0}$ \\
\hline 0.54 & 13938 & 326 & 136 & $\mathbf{0}$ \\
\hline 0.55 & 13854 & 406 & 140 & $\mathbf{0}$ \\
\hline 0.56 & 13806 & 424 & 170 & 0 \\
\hline 0.57 & 13774 & 458 & 168 & 0 \\
\hline 0.58 & 13794 & 410 & 196 & $\mathbf{0}$ \\
\hline 0.59 & 13816 & 432 & 152 & $\mathbf{0}$ \\
\hline 0.60 & 13836 & 402 & 162 & 0 \\
\hline 0.61 & 13810 & 423 & 164 & 3 \\
\hline 0.62 & 13678 & 528 & 186 & 8 \\
\hline 0.63 & 13336 & 796 & 254 & 14 \\
\hline 0.64 & 12794 & 1211 & 379 & 16 \\
\hline 0.65 & 12371 & 1479 & 523 & 27 \\
\hline 0.66 & 11911 & 1790 & 680 & 19 \\
\hline 0.67 & 11867 & 1802 & 705 & 26 \\
\hline
\end{tabular}




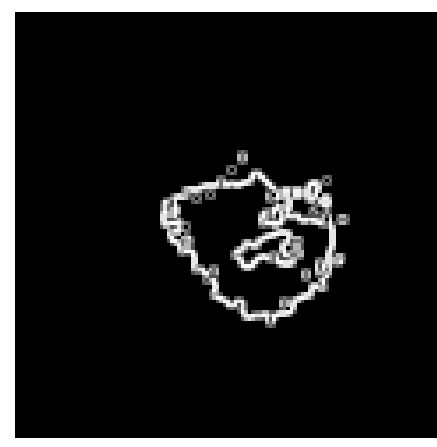

Fig. 2. Gradient Magnitude: 28J Impact, 0.57 Threshold.

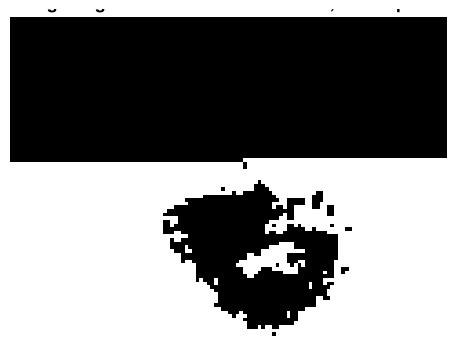

Fig. 3. Edge Segmentation: 28J Impact, 0.57 Threshold.

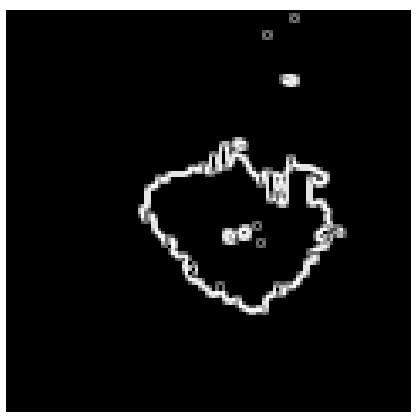

Fig. 4. Gradient Magnitude: 28J Impact, 0.60 Threshold.

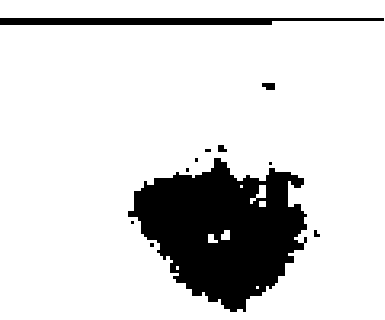

Fig. 5. Edge Segmentation: 28J Impact, 0.60 Threshold (Optimum Segmentation)

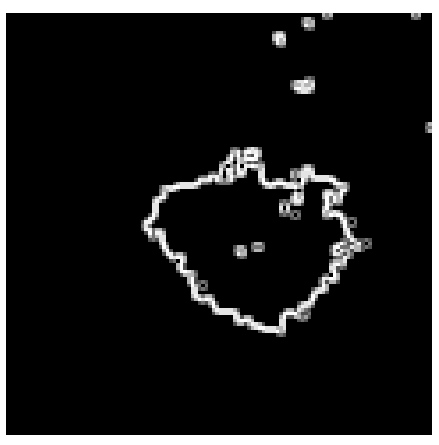

Fig. 6. Gradient Magnitude: 28J Impact, 0.61 Threshold.

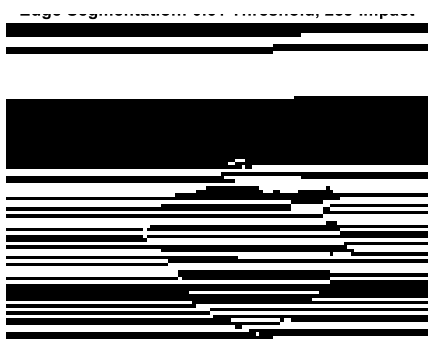

Fig. 7. Edge Segmentation: 28J Impact, 0.61 Threshold (No Segmentation Possible).

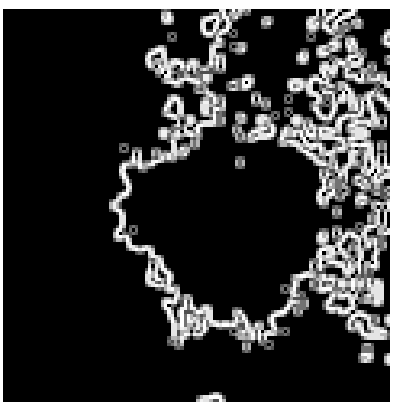

Fig. 8. Gradient Magnitude: 28J Impact, 0.67 Threshold.

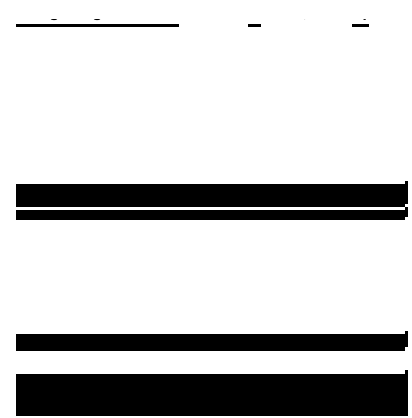

Fig. 9. Edge Segmentation: 28J Impact, 0.67 Threshold (No Segmentation Possible). 


\section{B. 42J Impact Energy}

Table II shows effect of varying the threshold level on the pixel distribution of an impact damaged RIM composite structure. The structure suffered a 42J of impact energy delivered through a vertically launched load, while Fig. 10-19 shows both Gradient Field and Segmentation images.

TABLE II. PIXEL DISTRIBUTION FOR 42J IMPACT

\begin{tabular}{|c|c|c|c|c|}
\hline Threshold & Level 0 & Level 128 & Level 180 & Level 255 \\
\hline 0.29 & 14400 & 0 & 0 & 0 \\
\hline 0.30 & 14388 & 12 & 0 & 0 \\
\hline 0.31 & 14356 & 32 & 12 & $\mathbf{0}$ \\
\hline 0.32 & 14290 & 76 & 34 & $\mathbf{0}$ \\
\hline 0.33 & 14186 & 158 & 56 & $\mathbf{0}$ \\
\hline 0.34 & 14126 & 186 & 88 & $\mathbf{0}$ \\
\hline 0.35 & 14134 & 164 & 102 & $\mathbf{0}$ \\
\hline 0.36 & 14138 & 166 & 96 & 0 \\
\hline 0.37 & 14150 & 162 & 88 & $\mathbf{0}$ \\
\hline 0.38 & 14132 & 184 & 84 & $\mathbf{0}$ \\
\hline 0.39 & 14134 & 170 & 96 & $\mathbf{0}$ \\
\hline 0.40 & 14060 & 224 & 116 & $\mathbf{0}$ \\
\hline 0.41 & 13990 & 282 & 128 & $\mathbf{0}$ \\
\hline 0.42 & 14002 & 260 & 138 & $\mathbf{0}$ \\
\hline 0.43 & 13982 & 282 & 128 & $\mathbf{0}$ \\
\hline 0.44 & 13984 & 278 & 138 & $\mathbf{0}$ \\
\hline 0.45 & 13976 & 292 & 132 & $\mathbf{0}$ \\
\hline 0.46 & 13950 & 292 & 158 & $\mathbf{0}$ \\
\hline 0.47 & 13912 & 332 & 156 & $\mathbf{0}$ \\
\hline 0.48 & 13894 & 344 & 162 & $\mathbf{0}$ \\
\hline 0.49 & 13910 & 324 & 166 & $\mathbf{0}$ \\
\hline 0.50 & 13912 & 316 & 172 & $\mathbf{0}$ \\
\hline 0.51 & 13874 & 338 & 188 & 0 \\
\hline 0.52 & 13828 & 382 & 190 & 0 \\
\hline 0.53 & 13758 & 402 & 238 & 2 \\
\hline 0.54 & 13702 & 465 & 230 & 3 \\
\hline 0.55 & 13587 & 524 & 283 & 6 \\
\hline 0.56 & 13445 & 644 & 307 & 4 \\
\hline 0.57 & 13129 & 850 & 415 & 6 \\
\hline 0.58 & 12871 & 1070 & 452 & 7 \\
\hline 0.59 & 12643 & 1201 & 533 & 23 \\
\hline 0.60 & 12583 & 1275 & 524 & 18 \\
\hline
\end{tabular}

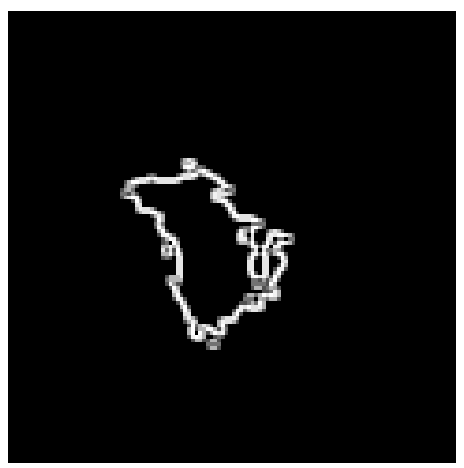

Fig. 10. Gradient Magnitude: 42J Impact, 0.41 Threshold.

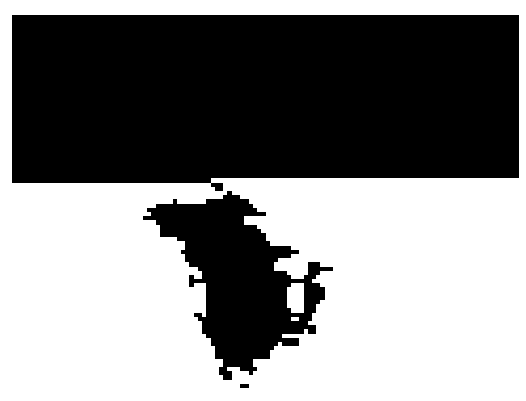

Fig. 11. Edge Segmentation: 42J Impact, 0.41 Threshold.

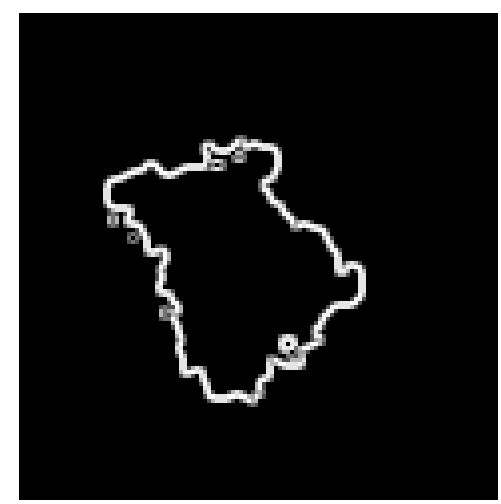

Fig. 12. Gradient Magnitude: 42J Impact, 0.48 Threshold.

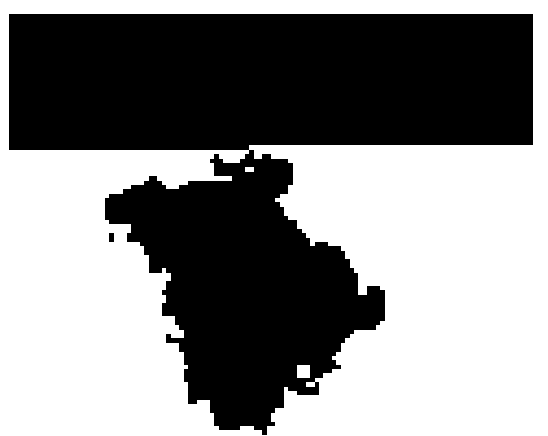

Fig. 13. Edge Segmentation: 42J Impact, 0.48 Threshold.

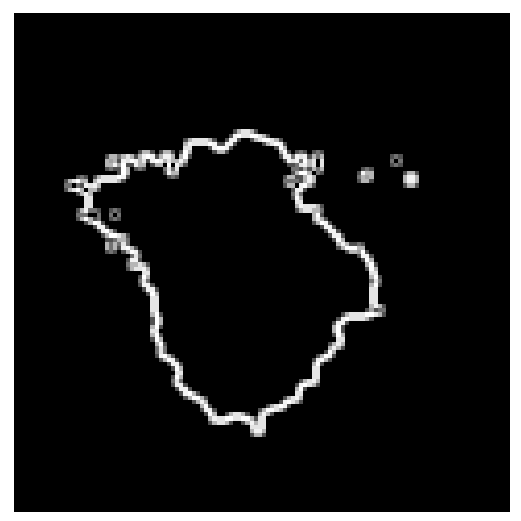

Fig. 14. Gradient Magnitude: 42J Impact, 0.52 Threshold. 


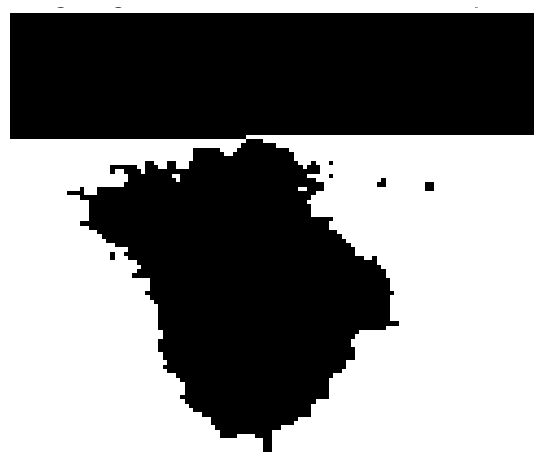

Fig. 15. Edge Segmentation: 42J Impact, 0.52 Threshold (Optimum Segmentation).

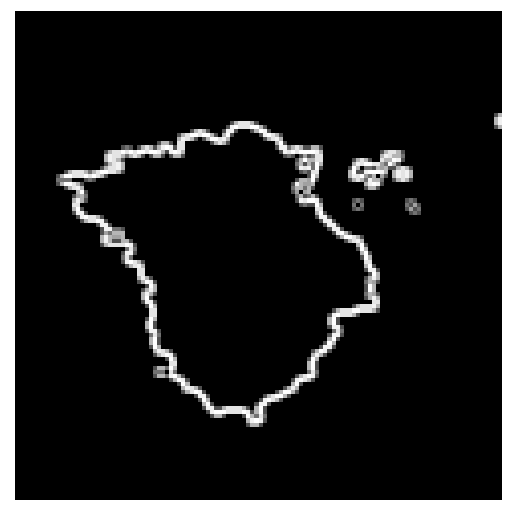

Fig. 16. Gradient Magnitude: 42J Impact, 0.53 Threshold.

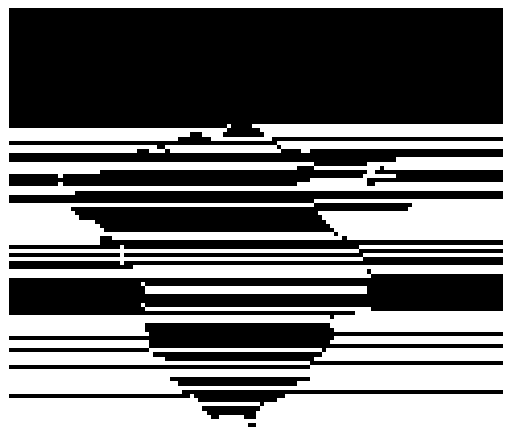

Fig. 17. Edge Segmentation 42J Impact, 0.53 Threshold (No Segmentation Possible).

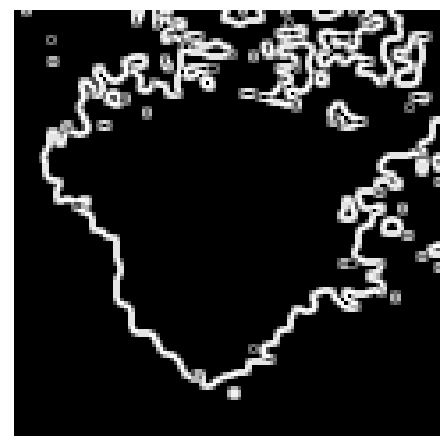

Fig. 18. Gradient Magnitude: 42J Impact, 0.60 Threshold.

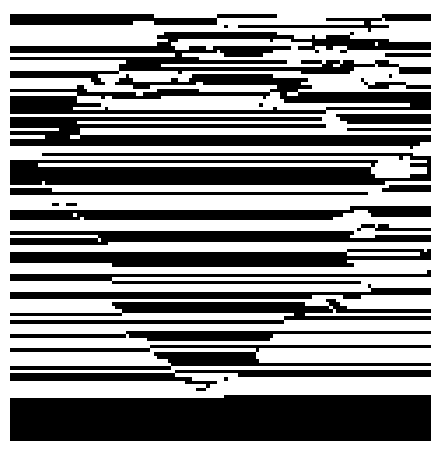

Fig. 19. Edge Segmentation 42J Impact, 0.60 Threshold (No Segmentation Possible).

Table III shows effect of varying the threshold level on the pixel distribution of an impact damaged RIM composite structure. The structure suffered a 56J of impact energy delivered through a vertically launched load, while Fig. 20-25 shows both Gradient Field and Segmentation images.

\section{56J Impact Energy}

TABLE III. PIXEL DistRIBUTION FOR 56J IMPACT

\begin{tabular}{|c|c|c|c|c|}
\hline Threshold & Level 0 & Level 128 & Level 180 & Level 255 \\
\hline 0.18 & 14400 & 0 & 0 & 0 \\
\hline 0.19 & 14392 & 7 & 0 & 1 \\
\hline 0.20 & 14340 & 48 & 3 & 9 \\
\hline 0.21 & 14226 & 147 & 8 & 19 \\
\hline 0.22 & 13936 & 365 & 60 & 39 \\
\hline 0.23 & 13686 & 518 & 161 & 35 \\
\hline 0.24 & 13360 & 799 & 197 & 44 \\
\hline 0.25 & 13138 & 961 & 250 & 51 \\
\hline 0.26 & 12675 & 1338 & 316 & 71 \\
\hline 0.27 & 12369 & 1552 & 407 & 72 \\
\hline 0.28 & 12063 & 1742 & 509 & 86 \\
\hline 0.29 & 11988 & 1821 & 516 & 75 \\
\hline 0.30 & 11816 & 2018 & 504 & 62 \\
\hline 0.31 & 11752 & 2062 & 530 & 56 \\
\hline 0.32 & 11780 & 1994 & 575 & 51 \\
\hline 0.33 & 11778 & 2062 & 523 & 37 \\
\hline 0.34 & 11766 & 2131 & 472 & 31 \\
\hline 0.35 & 11732 & 2127 & 506 & 35 \\
\hline 0.36 & 11640 & 2181 & 536 & 43 \\
\hline 0.37 & 11544 & 2200 & 618 & 38 \\
\hline 0.38 & 11660 & 2082 & 622 & 36 \\
\hline 0.39 & 11856 & 1924 & 581 & 39 \\
\hline 0.40 & 12202 & 1710 & 464 & 24 \\
\hline 0.41 & 12416 & 1570 & 396 & 18 \\
\hline 0.42 & 12708 & 1355 & 328 & 9 \\
\hline 0.43 & 12877 & 1188 & 329 & 6 \\
\hline 0.44 & 13003 & 1108 & 282 & 7 \\
\hline 0.45 & 13095 & 1015 & 288 & 2 \\
\hline 0.46 & 13165 & 933 & 299 & 3 \\
\hline 0.47 & 13277 & 884 & 286 & 3 \\
\hline 0.48 & 13357 & 825 & 216 & 2 \\
\hline 0.49 & 13507 & 714 & 178 & 1 \\
\hline 0.50 & 13661 & 564 & 173 & 2 \\
\hline 0.51 & 13721 & 524 & 155 & 0 \\
\hline 0.52 & 13757 & 514 & 129 & $\mathbf{0}$ \\
\hline $\begin{array}{l}0.53 \\
\end{array}$ & 13852 & 464 & 82 & 2 \\
\hline
\end{tabular}




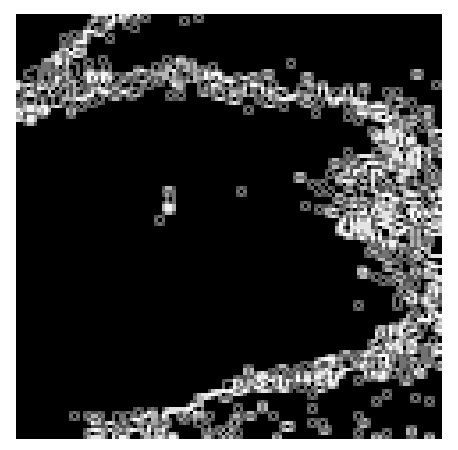

Fig. 20. Gradient Magnitude: 56J Impact, 0.31 Threshold.

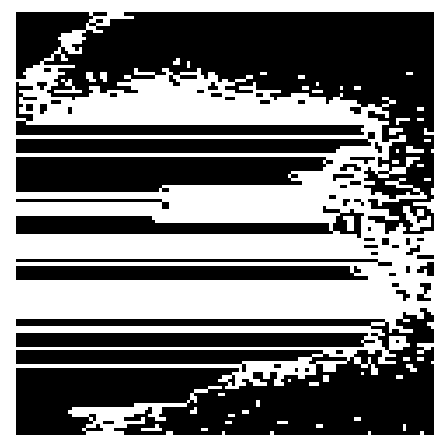

Fig. 21. Edge Segmentation 56J Impact, 0.31 Threshold (No Segmentation Possible).

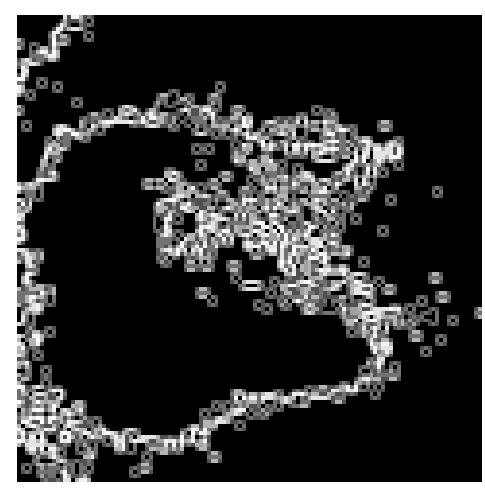

Fig. 22. Gradient Magnitude: 56J Impact, 0.37 Threshold.

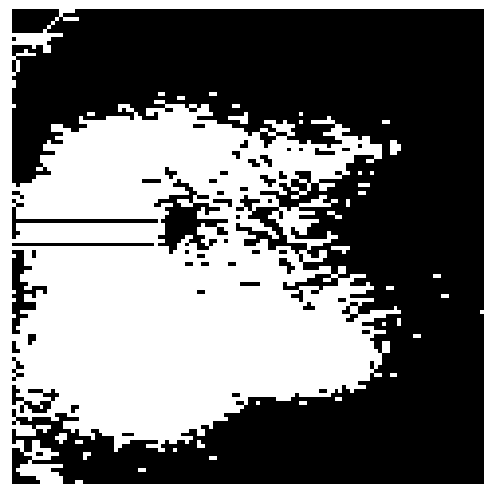

Fig. 23. Edge Segmentation 42J Impact, 0.37 Threshold (No Segmentation Possible).

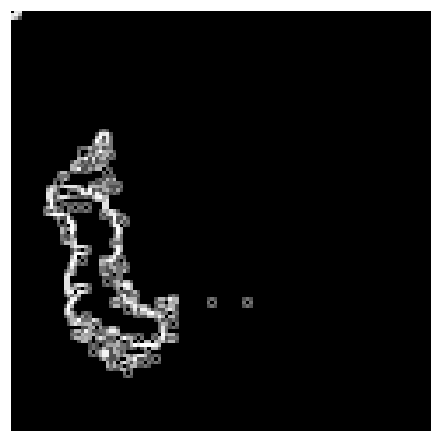

Fig. 24. Gradient Magnitude: 56J Impact, 0.51 Threshold.

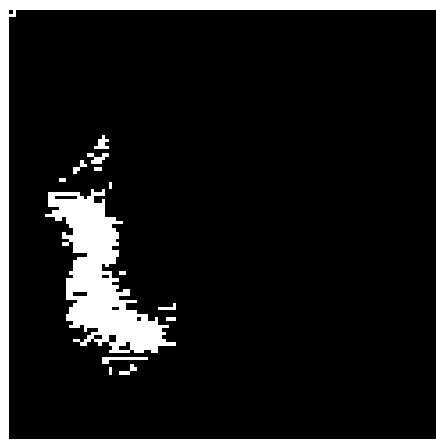

Fig. 25. Edge Segmentation 42J Impact, 0.51 Threshold (No Segmentation Possible)

\section{ANALYSIS AND DISCUSSION}

Fig. 26 shows the used system for impact damage analysis, while Tables I to III and Fig. 2 to 25 show the resulting gradient and segmented images.

Classification and determination of optimum threshold value that uncover damage in relation to the extent of impact energy, is computed as a result of correlating gradient field images with segmented images. $\mathrm{L}=255$ is regarded as the critical Gray level that determines the optimum threshold value.

Three levels of impact energies applied to the RIM composite structure. The results can be interpreted as follows:

\section{A. 28J Impact Energy}

Features of the damaged area started to appear at threshold value of 0.43 , with optimum features at threshold value of 0.60 . The damaged area stated to merge with the surrounded areas at threshold value of 0.61 with total merge at threshold value of 0.67 .

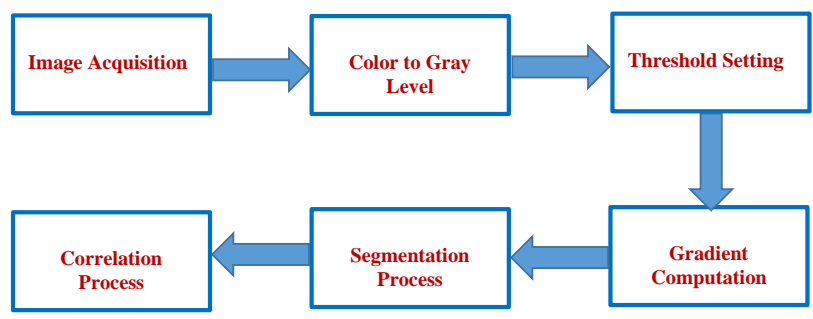

Fig. 26. Image processing system 
Four sampled images are presented, which cover two intervals of pixel population:

- Two sampled images: $(\mathrm{L}=255)$ empty.

- Two sampled images: $(\mathrm{L}=255)$ populated

The sampling resulted in the following pairs of correlated images as shown the respective figures: \{(Fig. 2, Fig. 3), (Fig. 4, Fig. 5), (Fig. 6, Fig. 7), Fig. 8, Fig. 9)\}.

The correlation resulted in one optimum threshold value for an impact energy of $28 \mathrm{~J}$, which is 0.60 . The algorithm will take this value as a second parameter and correlate it to the impact energy. Hence, a new pair is resulted, $\{28 \mathrm{~J}, 0.6\}$.

From Table I, data string describing the level of damage at $28 \mathrm{~J}$ is obtained by correlating impact energy level, threshold level and pixel population, thus obtaining: $\{28 \mathrm{~J}, 0.6,1,13836$, $402,162,0\}$

A value of 1 is added to indicate that the damage is not severe as segmentation is achieved.

\section{B. 42J Impact Energy}

Features of the damaged area started to appear at threshold value of 0.30 , with optimum features at threshold value of 0.52 . The damaged area stated to merge with the surrounded areas at threshold value of 0.53 with total merge at threshold value of 0.60 .

In comparison with the case of $28 \mathrm{~J}$ impact, it is found (as expected) that the threshold values are lower in as the impact energy is increased from $28 \mathrm{~J}$ to $42 \mathrm{~J}$ by a $14 \mathrm{~J}$ magnitude. This indicates that a larger impact energy requires lower threshold to uncover its extent of damage.

Five sampled images are presented, which cover two intervals of pixel population:

- Three sampled images: $(\mathrm{L}=255)$ empty.

- Two sampled images: $(\mathrm{L}=255)$ populated

The sampling resulted in the following pairs of correlated images as shown in the respective figures: $\{$ (Fig. 10, Fig. 11), (Fig. 12, Fig. 13), (Fig. 14, Fig. 15), Fig. 16, Fig. 17), (Fig. 18, Fig. 19)\}.

The correlation resulted in one optimum threshold value for an impact energy of 42J, which is 0.52 . This also a lower value compared to the $28 \mathrm{~J}$ case of 0.6 . The algorithm will take this value as a second parameter and correlate it to the impact energy. Hence, a new pair is resulted, \{42J, 0.52\}.

From Table II, data string describing the level of damage at $28 \mathrm{~J}$ is obtained by correlating impact energy level, threshold level and pixel population, thus obtaining:

A value of 1 is added to indicate that the damage is not severe as segmentation is achieved $\{42 \mathrm{~J}, 0.52,1,13828,382$, $190,0\}$.

\section{56J Impact Energy:}

At 56J impact energy, the pixel distribution and population characteristics and threshold values did not follow the previous models for both $28 \mathrm{~J}$, and $42 \mathrm{~J}$ impact energies as the tested composite suffered fiber pull out and fiber extraction, which unbalanced the fiber-matrix relationship in terms of response to external scanning sources.

Three sampled images are presented, but could not confine the samples to the previous set intervals due to the severe damage and fiber breakage. Also, segmentation algorithm failed to isolate damaged region(s) as the whole component suffered total damage.

The sampling resulted in the following pairs of correlated images as shown in the respective figures: $\{$ (Fig. 20, Fig. 21), (Fig. 22, Fig. 23), (Fig. 24, Fig. 25)

The correlation resulted in no single optimum threshold value for an impact energy of 56J possible.

From Table III, data string describing the level of damage at $56 \mathrm{~J}$ is obtained by correlating impact energy level, threshold level, in this case a 0 is also added (to indicate severe damage) in addition to a selected threshold value (equivalent to optimum threshold in non-severe damage cases) at which most of the damaged area appear (in this case 0.37) as there is no correlation due to no segmentation, thus obtaining: $\{56 \mathrm{~J}, 0.37$, $0,11544,2200,618,38\}$

It is clear from the pixel population that the pixel distribution pattern is markedly different when severe damage occurs.

Fig. 27 to 29 show histograms obtained for three impact energies. From the histograms, it is clear that at 56J impact, a dramatic change occurs in the tested composite structure, which is fiber breakage and pull out. Thus affecting pixel values and distribution pattern, and resulting in a unique statistical accumulation.

The obtained histogram results correlates well with gradient contours shown in Fig. 30-32, where at 56J different characteristics are presented with the effect of impact is more marked and no evidence of localized damage as in the cases of 28J and 42J impact energy levels.

Fig. 33-35 illustrate the relationships covering three important factors:

- Impact Energy (IE)

- Affected or Damaged Region(s) (Gradient Field Area) (GFA)

- Threshold Value (TH)

Fig. 33 presents a direct relationship between Impact Energy and Gradient Field Area. This is logical as the higher the impact energy the larger the affected area.

Fig. 34 presents an inverse relationship between the selected Threshold and Gradient Field Area. This is also logical, since the more the affected area, the less of Threshold Value is required to uncover the affected region boundaries. This is also consistent with increasing the Impact Energy.

Fig. 35 establishes the relationship between Impact Energy and Gradient Field Area, which is a direct relationship as deduced from Fig. 34. 


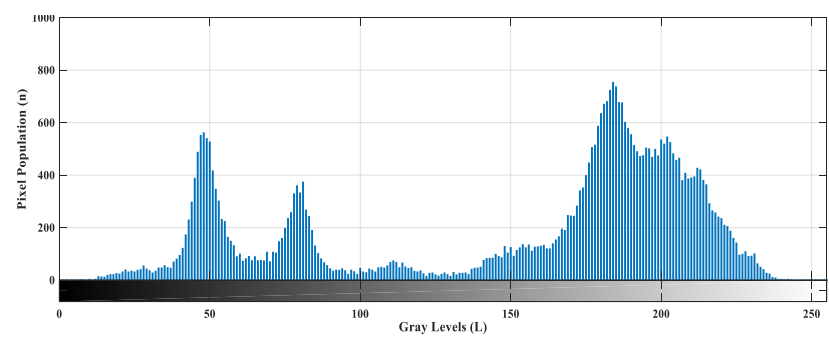

Fig. 27. Image Histogram: 28J Impact, 0.60 Threshold.

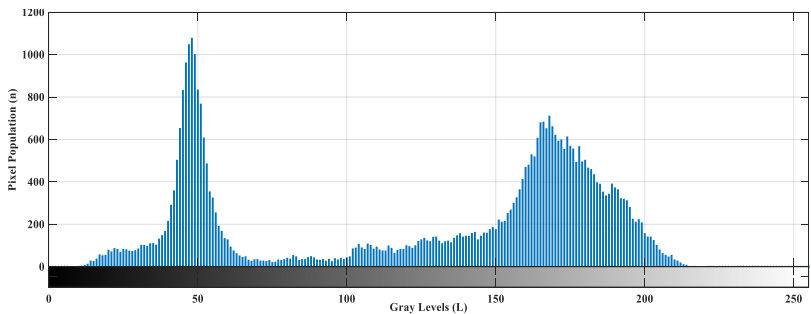

Fig. 28. Image Histogram: 42J Impact, 0.52 Threshold.

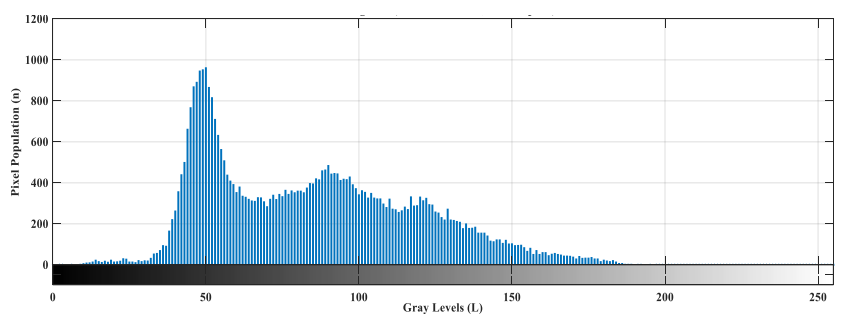

Fig. 29. Image Histogram: 56J Impact, 0.37 Threshold.

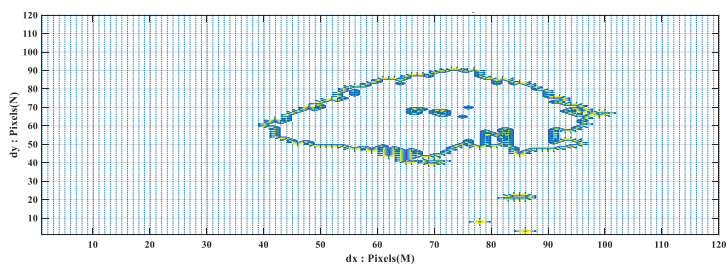

Fig. 30. Gradient Field Contour: 28J Impact, 0.60 Threshold.

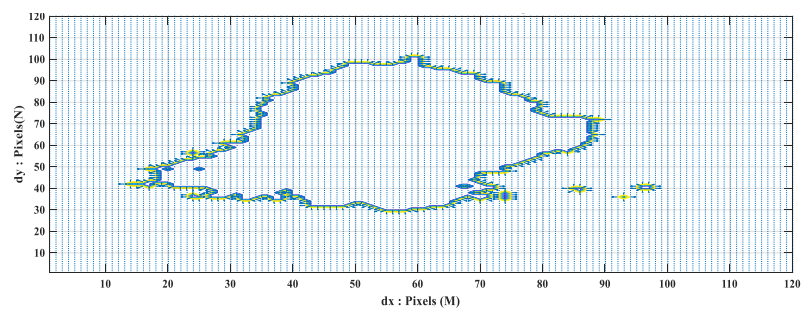

Fig. 31. Gradient Field Contour: 42J Impact, 0.52 Threshold.

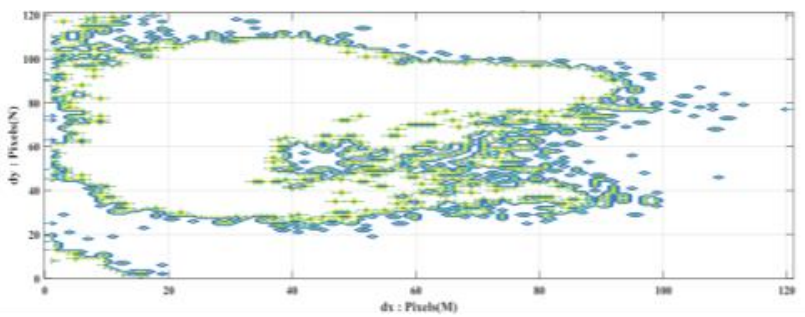

Fig. 32. Gradient Field Contour: 56J Impact, 0.37 Threshold.

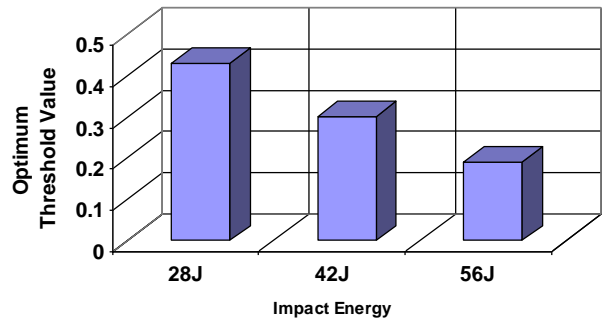

Fig. 33. Relationship between Impact Energy and Selected Threshold.

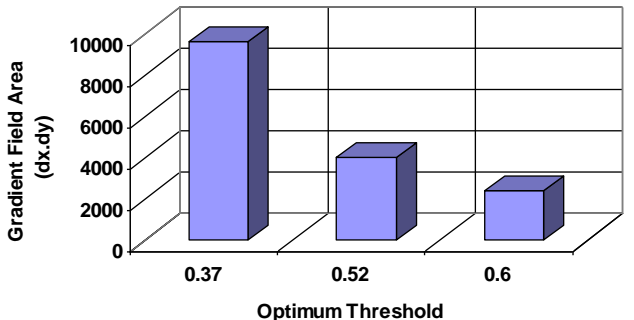

Fig. 34. Relationship between Optimum Threshold and Gradient Field Area.

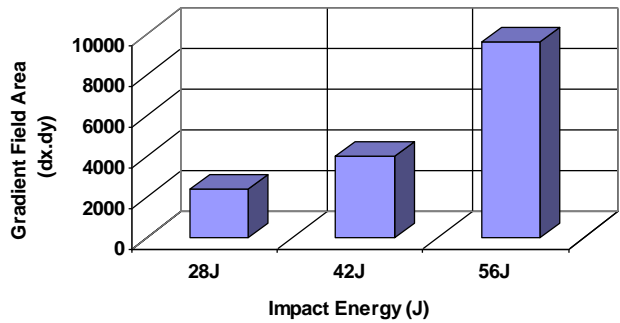

Fig. 35. Relationship between Impact Energy and Gradient Field Area.

The obtained images consist of two parts as shown in equation (1):

$\operatorname{Im}$ age $=\sum_{L=0}^{255}$ Healthy Part $($ Threshold $)+\sum_{L=0}^{255}$ Damaged Part (Threshold $)$.

Equation (1) can be further represented in relation to threshold values as shown in equation (2), which also applies to undamaged composites

$$
\text { Im age }=\left[\sum_{\text {Threshold }=0}^{1.0}\left(L_{0}+L_{128}+L_{180}\right)\right]+\left[L_{255}\right]
$$

Applying equation (2) to the tested composites at impact energies of $28 \mathrm{~J}, 42 \mathrm{~J}$, and $56 \mathrm{~J}$, result in equations (3), (4), and (5).

$$
\begin{aligned}
& \text { Image }(28 J)=\left[\sum_{\text {Threshold }=0}^{0.6}\left(L_{0}+L_{128}+L_{180}\right)\right] . \\
& \operatorname{Im} \text { age }(42 J)=\left[\sum_{\text {Threshold }=0}^{0.52}\left(L_{0}+L_{128}+L_{180}\right)\right] . \\
& \operatorname{Im} \text { age }(56 J)=\left[\sum_{\text {Threshold }=0}^{0.37}\left(L_{0}+L_{128}+L_{180}\right)\right]+\left[L_{255}\right]
\end{aligned}
$$




\section{CONCLUSIONS}

From the previous observations, we can establish the following:

1) Very Low Impact Energy ( $0 J \leq I E \leq 28 J$

No observed damage.

2) Lower Impact Energies ( $28 J \leq I E \leq 42 J)$ :

a) Similar Histogram Characteristics

b) Localized Damage

c) Threshold decreases as Impact Energy Increases Increases.

d) Gradient Field Area Increases as Impact Energy

3) Higher Impact Energies ( $I E \geq 56 J$ )

a) Unique Histogram Characteristics

b) Non-Localized Damage Increases

c) Threshold decreases dramatically as Impact Energy

d) Gradient Field Area Increases markedly as Impact Energy Increases.

The Optimum Threshold level that is used to uncover the extent of damage is related to Impact Energy through expression (6):

$T h_{\text {Optimum }}=K *[\log (I E)]^{-1}$

$T h_{\text {Optimum }}$ is bounded by the following conditions, which are established experimentally and is a function of sample mechanical properties as well as used imaging technique:

a) Very Low Impact Energies ( $0 \mathrm{~J}<\mathrm{IE}<28 \mathrm{~J})$ : $\mathrm{K}=0.5$

b) Lower Impact Energies ( $28 J \leq I E \leq 42 J)$ : $\mathrm{K}=2$

c) Higher Impact Energies ( $I E \geq 56 J$ ): $\mathrm{K}=1.5$

From Equation (1), we can reproduce a guiding curve describing the relationship between Required Optimum Threshold to uncover damage level and Impact Energy, as shown in Fig. 36.

From the plot, the three main bounding conditions are clear:

1) At very low Impact Energies, the composite sample will have negligible damage, hence, the sample and surface will possess uniform structure and that reduces the Optimum Threshold value to a very low level. Such Levels of Impact Energies can be neglected as it will not affect component safety and reliability.

2) At Lower Impact Energies, there is and evidence of damage and surface deformation as the Optimum Threshold increases.

3) At Higher Impact Energies, most of the tested component is damage as the damage propagates both in depth and breadth. This is a critical case of damage.

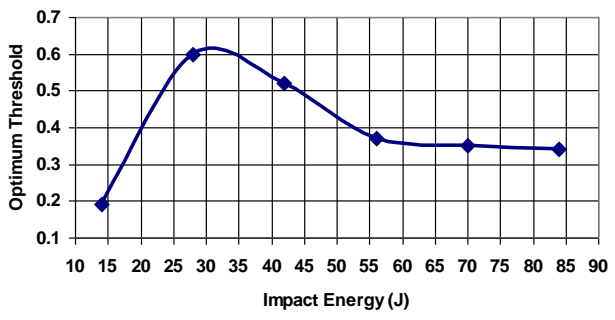

Fig. 36. Relationship between Impact Energy Optimum Threshold.

The proposed approach proved to be simpler, yet very effective approach for separating and segmenting damaged areas in composite structures compared to traditional techniques.

\section{REFERENCES}

[1] Yue El-Hage, Simon Hind, and Francois Robitaille. 2018. Thermal conductivity of textile reinforcements for composites, Journal of Textiles and Fibrous Materials, I :1-12. DOI: $10.1177 / 2515221117751154$

[2] Francesco Ciampa, Pooya Mahmoodi, Fulvio Pinto, and Michele Meo. 2018. Recent Advances in Active Infrared Thermography for NonDestructive Testing of Aerospace Components. Sensors, 609: 1-37. DOI: $10.3390 / \mathrm{s} 18020609$

[3] Carlos G Dávila and Chiara Bisagni. 2018. Fatigue life and damage toleranc of postbuckled composite stiffened structures with indentation damage. Journal of Composite Materials, 52(7): 931-943. DOI: 10.1177/0021998317715785.

[4] L. Ma and D. Liu. 2016. Delamination and fiber-bridging damage analysis of angle-ply laminates subjected to transverse loading. International Journal of Imaging Systems and Technology, 50 (22): 3063-3075. DOI: 10.1177/0021998315615647.

[5] F.Wang, J. Liu, Y. Liu, and Y. Wan. 2016. Research on the fiber lay-up orientation detection of unidirectional CFRP laminates composite using thermal-wave radar imaging. International Journal of Imaging Systems and Technology, 84: 54-66. http://dx.doi.org/10.1016/j.ndteint .2016.08.002

[6] T.Liang, W. Ren, G. Tian, M. Elradi, Y. Gao. 2016. Low energy impact damage detection in CFRP using eddy current pulsed thermography. Composite Structure, 143: 352-361. DOI: 10.1016/j.compstruct. 2016.02.039.

[7] R.Yang, Y. He, H. Zhang. 2016. Progress and trends in nondestructive testing and evaluation for wind turbine composite blade. Renewable and Sustainable Energy Reviews, 60: 1225-1250. DOI: 10.1016/ j.rser.2016.02.026.

[8] L. Ma and D. Liu. 2016. Delamination and fiber-bridging damage analysis of angle-ply laminates subjected to transverse loading. International Journal of Imaging Systems and Technology. 50: 30633075. DOI: $10.1177 / 0021998315615647$

[9] F.Wang, J. Liu, Y. Liu, and Y. Wang. 2016. Research on the fiber lay-up orientation detection of unidirectional CFRP laminates composite using thermal-wave radar imaging. International Journal of Imaging Systems and Technology. 84: 54-66. DOI: 10.1016/j.ndteint.2016.08.002

[10] T.Liang, W. Ren, G. Tian, M. Elradi, Y. Gao. 2016. Low energy impact damage detection in CFRP using eddy current pulsed thermography. Composite Structure, 143:352-361. DOI: 10.1016/j.compstruct.2016.02.039.

[11] M. Iskandarani. 2017. Correlating and Modeling of Extracted Features from PVT Images of Composites using Optical Flow Technique and Weight Elimination Algorithm Optimization [OFT-WEA]. Journal of Computer Science, 13(9): 371-379. DOI: 10.3844/jcssp.2017.371.379

[12] F.Peng, J. Li, and M. Long. 2015. Identification of Natural Images and Computer-Generated Graphics Based on Statistical and Textural Features. Journal of Forensic Sciences, 60 (2): 435-443. DOI: 10.1111/1556-4029. 
[13] P. Geng, X. Su, T. Xu. J. Liu. 2016. Multi-modal Medical Image Fusion Based on the Multiwavelet and Non sub sampled Direction Filter Bank. International Journal of Signal Processing, Image Processing and Pattern Recognition, 8(11):75-84. DOI: 10.14257/ijsip.2015.8.11.08

[14] K. Santhi, R. WahidaBanu. 2015. Adaptive contrast enhancement using modified histogram equalization. Optik - International Journal for Light and Electron Optics. Elsevier, 126: 1809-1814. DOI: 10.1016/ j.ijleo.2015.05.023

[15] L. Huang,W. Zhao, Z. Sunand J. Wang. 2015. An advanced gradient histogram and its application for contrast and gradient enhancement. Optik - International Journal for Light and Electron Optics. Elsevier, 31: 86 - 100. DOI: 10.1016/j.jvcir.2015.06.007

[16] H. Xu, Q. Chen, C. Zuo, C. Yang and N. Liu. 2015. Range limited double-thresholds multi-histogram equalization for image contrast enhancement. Optical Review. Springer, 22: 246 - 255. DOI: 10.1016/j.ijleo.2011.12.057

[17] B.Ashwini, B. Yuvaraju. 2016. Feature Extraction Techniques for Video Processing in MATLAB. International Journal of Innovative Research in Computer and Communication Engineering, 4 (4): 5292-5296. DOI: 10.15680/IJIRCCE.2016. 0404223.

[18] M.Iskandarani.2018. Characterization of Composite Structure Surface Uniformity using Interval Based Gradient Field Histogram Analysis of Thermographic Images (IGF-HA). Journal of Computer Science, 14(6): 816-828. DOI: $10.3844 /$ jcssp.2018.819.828.

[19] M.Iskandarani. 2018. Modelling of Thermal Storage in Damaged Composite Structures using Time Displaced Gradient Field Technique (TDGF). (IJACSA) International Journal of Advanced Computer Science and Applications, 9(6): 55-59. DOI: 10.14569/IJACSA.2018.090608 